\title{
An Iris Detection Method Based on Structure Information
}

\author{
Jiali Cui ${ }^{1}$, Tieniu Tan $^{1+}$, Xinwen Hou ${ }^{1}$, Yunhong Wang ${ }^{1,2}$, Zhuoshi Wei ${ }^{1}$ \\ ${ }^{1}$ Center for Biometrics and Security Research \\ National Laboratory of Pattern Recognition, Institute of Automation, \\ Chinese Academy of Sciences, P.O. Box 2728, Beijing, P.R.China, 100080 \\ \{jlcui, tnt, xwhou, wangyh, zswei\} @nlpr.ia.ac.cn \\ ${ }^{2}$ School of Computer Science and Engineering \\ Beihang University, Beijing, 100083, P. R. China \\ yhwang@buaa.edu.cn
}

\begin{abstract}
In this paper, the authors propose an iris detection method to determine iris existence. The method extracts 4 types of features, i.e., contrast feature, symmetric feature, isotropy feature and disconnected feature. Adaboost is adopted to combine these features to build a strong cascaded classifier. Experiments show that the performance of the method is promising in terms of high speed, accuracy and device independence. ...
\end{abstract}

\section{Introduction}

Currently, due to the development of the networked society, biometrics [1] has become a promising topic in the last decades and has many applications such as access control, network security, etc. Iris recognition, as a typical biometric technique, has been receiving more and more attention [2-5] due to its high accuracy and matching speed. The history of iris recognition comes back to the $19^{\text {th }}$ century, when a French doctor recognized prisoners by their iris color. In the last decade, many researchers were interested in it. Daugman realized an automatic recognition system in 1993 and its identification accuracy is 100\% [2]. Wildes developed a device to get iris images from distance and used super-resolution method to obtain clear images [3]. Previous work [4, 5] used wavelet and Gabor filters to extract iris features, respectively.

In our system, iris detection is an important procedure in preprocessing. We must emphasize that the term iris detection in this paper is defined as determining whether there is an iris in an input image. Although 'iris detection' has been used in other papers, its meaning is similar to eye detection for gaze evaluation, expression synthesis, face recognition, etc. Iris detection is a necessary procedure in an iris recognition system and its need comes mainly from the following reasons. First, it can improve the performance of iris recognition systems. Most of the systems assume

+ Corresponding author. Tel: 86-10-62616658, Fax: 86-10-62551993, Email: tnt@nlpr.ia.ac.cn. 
that there is an iris in the input image. Since many images in the input sequence of iris sensor do not include iris, it is very necessary to differ iris images (images including iris) from non-iris images (images not including iris) to avoid unnecessary computation to save time. It can also avoid matches between non-iris images and iris templates to give wrong decision. Sometimes, these non-iris images cannot be excluded by high-light blocks, circle verification and liveness detection. Another reason is that it is a general and fast coarse localization method and important for iris recognition. As we know, many iris localization methods, such as Daugman's circlefinding method, take a coarse-to-fine strategy. Then, the speed and accuracy of iris localization is influenced by coarse localization results. Moreover, it is a method independent of device. Some systems find high-light blocks as coarse localization, however, the number and size of high-light blocks is different from device to device. So, a general coarse localization method needs no change when device changes.

However, in real time system, there seems no fast iris detection method up to now. Although our previous work [6] detects iris using an appearance-based method, it is not fast. Inspired by work [7], the authors propose a fast iris detection method based on structure information. The algorithm extracts 4 types of features, namely contrast feature, symmetric feature, isotropy feature and disconnected feature. Then Adaboost is adopted to select useful features from the feature set to build a cascaded classifier.

The remainder of the paper is organized as follows. The algorithm of iris detection to be proposed is described in detail in section 2. Section 3 gives the experimental results of the method. The last part of the paper concludes the whole paper.

\section{Proposed Method}

The tasks of iris detection are to determine iris existence in an image and, if existence, to locate its position and size. To fulfill these tasks, three major processes are necessary. The first process is iris modeling, which characterizes the iris structure properties. The second is feature extraction, which extracts iris features to represent iris structure. The last is feature selection and classifier design, which computes the classification ability of each feature and combines the features of good classification ability to build a classifier.

\subsection{Iris Modeling}

Iris modeling is to character iris properties and provide clues to feature extraction. To most of our knowledge, although color is important for iris patterns of westerners, most personal identification systems based on iris recognition use intensity images. Moreover, gray value of iris images captured via different devices is different. Although texture plays an important role, texture feature extraction is computational complex. So structure (gray level distribution), is most important for iris modeling.

If an iris image is seen as a surface, it can be found that iris (include pupil) is like an inverse hat (See Fig.1). Thus, an inverse-hat model is adopted. It indicates that the center of an iris is of low gray value and the surrounding parts are of high gray value. 


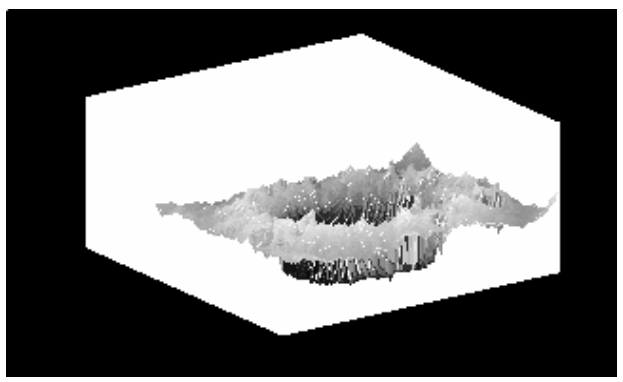

Fig.1. Inverse-hat model

\subsection{Feature Extraction}

Feature extraction is to extract quantized features to represent iris patterns. As we know, spatial gray level co-occurrence matrix (GLCM) is a widely used feature for structural patterns; however, GLCM is a statistical feature and computational complex. Based on the proposed inverse-hat model, four types of features are extracted and introduced as follows.

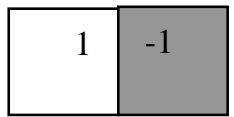

(1)

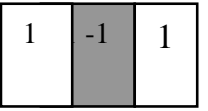

B.

(5)

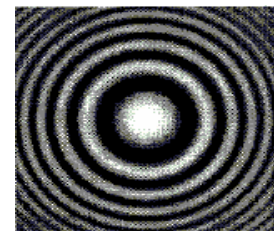

C. (9)

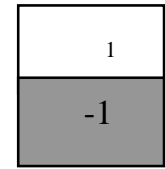

(2)

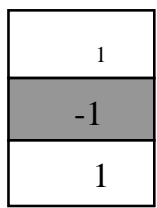

(6)

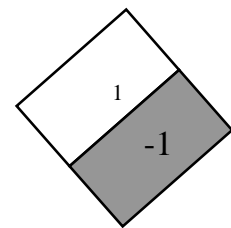

(3)

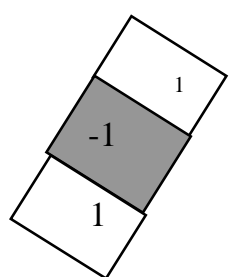

(7)

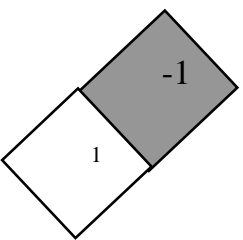

(4)

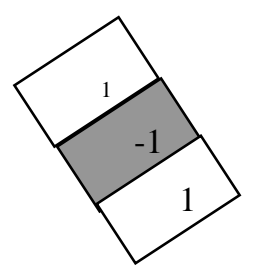

(8)

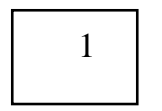

$-1$

D. (10)

Fig.2. Four types of features 
In Fig.2, four types of features are contrast feature (denoted by A, including 1, 2, 3, 4), symmetric feature (denoted by B, including 5, 6, 7, 8), isotropy feature (denoted by C, including 9) and disconnected feature (denoted by D, including 10), respectively. They are computed as follows.

The feature, denoted by $f$, is the difference $(d)$ of two regions normalized by the variance $(v)$ of the total image and is computed as follows.

(1) Compute $d$. For type A, B and D, $d=$ (sum of gray values in region denoted by 1 ) - (sum of gray values in region denoted by -1 ). For type $C, d$ is difference of two rings and computed as follows.

$$
\begin{aligned}
& s_{i}=\int_{r_{i}}^{r_{i+1}} \int_{0}^{2 \pi} I(\rho, \theta) d \rho d \theta \\
& d_{i, j}=s_{i}-S_{j}
\end{aligned}
$$

Circle generation used in Eqn. (1) is computed using Bresenham algorithm (a famous method in computer graphics).

(2) Computer the variance $v$ of the image. To obtain high speed, we use a short session to introduce a fast algorithm for variance computation. Let $h(i)$ be the histogram of the images and I be an image, then

$$
\begin{aligned}
& \operatorname{Var}(I)=E(I)^{2}-(E I)^{2} \\
& =\frac{1}{N} \sum_{i, j} I(i, j)^{2}-\left(\frac{1}{N} \sum_{i, j} I(i, j)\right)^{2} \\
& =\frac{1}{N} \sum_{i=1}^{255} h(i) i^{2}-\frac{1}{N^{2}}\left(\sum_{i, j} I(i, j)\right)^{2}
\end{aligned}
$$

Because $i^{2}$ ( $i=0,1, \ldots, 255$ ) can be made a lookup table, after carefully computation, it needs, at most, only $2 * \mathrm{M} * \mathrm{~N}+256$ integer additions, 256 integer multiplications and 2 divisions. Here $\mathrm{M}$ and $\mathrm{N}$ are the size of an image.

(3) The feature is $f=d / v$.

Finally, a feature set is built though changing the size of the regions shown in Fig.2.

\subsection{Feature Selection and Classifier Design}

The proposed method is inspired by Viola's work [7], which obtains high speed. Therefore, Adaboost is adopted to select features to build a cascaded classifier. Feature selection procedure based on Adaboost is introduced briefly because it is not the focus of the paper. Adaboost takes a rejection strategy to eliminate negative samples and put big weight on the outlier step by step (or layer by layer). If a sample passes all the layers, it can be seen as a positive sample. Its procedures are shown in Fig.3. 


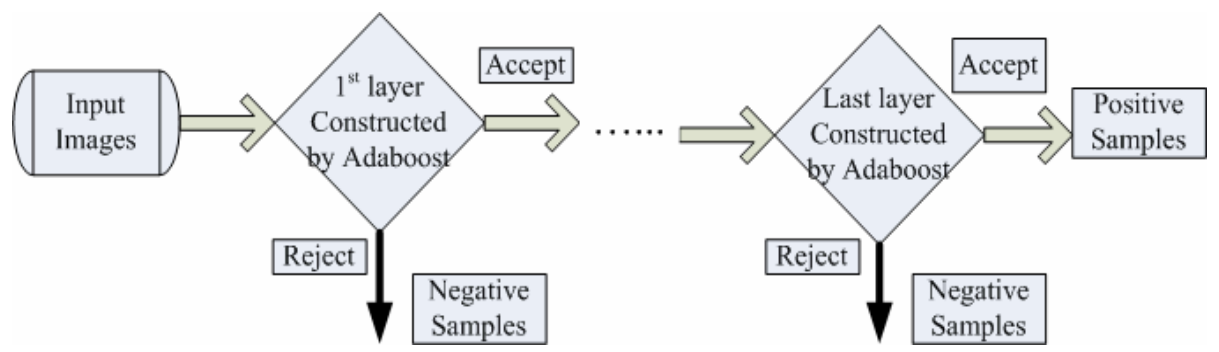

Fig. 3. Flowchart of iris detection

\section{Experimental Results and Analysis}

\subsection{Computer Configuration}

Here, computer configuration is provided to evaluate qualitative performance of the proposed method. Experiments are done on a PC with PIV 2.4GHz processor and 256M DRAM. The code is implemented with VC++6.0.

\subsection{Training Database}

The training database comprises of 12735 positive samples and 13924 negative samples. Positive samples include segmented iris images with rotation, scale and transition transform. We have two iris sensors denoted by sensor 1 and 2, respectively. Although samples based on different sensors is available, only one database (captured by sensor 1) is used in training process and the reason is that we want to verify the extension power of our method. Negative samples include 3 categories, namely natural images (1700 images including cars, persons, vessels, scenes, animals, etc and a database used for image retrieval is adopted here), face images (7035 images including only closed eyes) and background images (5189 images of the application background, such as wall, device, etc). Three categories negative samples are selected to make the method both general and specific. More face images with closed eyes are selected because those images are very similar to iris images and they occur very frequently in real application. Some examples of the training database are shown in Fig.4.

\subsection{Test Database}

Test database include 3000 iris images from 100 subjects and 3135 non-iris images. Iris images include 3 categories, (A) 1000 images captured by homemade sensor 1 , (B) 1000 images captured by homemade sensor 2, (C) 1000 images captured by either 
sensor 1 or 2, however, users wear glasses in acquisition. There is no overlap between training database and test database.
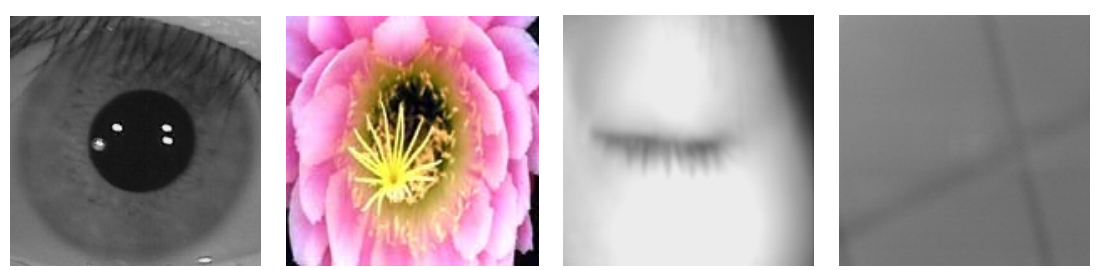

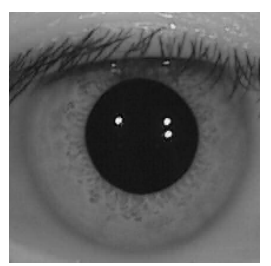

(a)

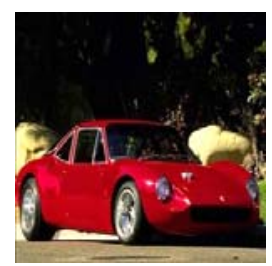

(b)

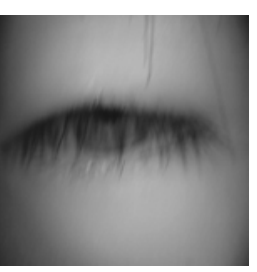

(c)

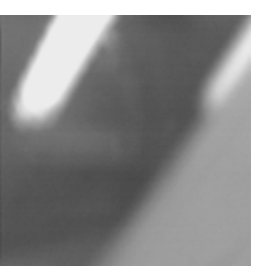

(d)

Fig. 4. Examples from training database (a) positive samples (b) natural images (c) face images with closed eyes (d) background images

\subsection{Results and Analysis}

In training process, a cascaded classifier with 24 layers (totally 437 weak classifiers) is obtained. The number of each feature is listed in Table 1.

Table 1. Number of each feature

\begin{tabular}{|l|l|l|l|l|l|l|l|l|l|l|}
\hline Feature Index. & 1 & 2 & 3 & 4 & 5 & 6 & 7 & 8 & 9 & 10 \\
\hline Number & 18 & 62 & 32 & 36 & 87 & 104 & 20 & 21 & 30 & 27 \\
\hline
\end{tabular}

It can be seen that feature 6 is selected 104 times and it indicates that vertical symmetry is very important to characterize iris structure.

Experiments also show that the method has promising performance in terms of speed and accuracy. Averagely, the detection process costs only $16 \mathrm{~ms}$. High speed makes it possible to search in an image exhaustively. The detection accuracy is listed in Table 2.

Table 2. Detection accuracy

\begin{tabular}{|c|c|c|}
\hline Category & $\begin{array}{c}\text { \# of Correctly classified } \\
\text { images }\end{array}$ & Recognition Rate \\
\hline Non-iris Images & 3087 & $98.4 \%$ \\
\hline Iris Images (A) & 980 & $98 \%$ \\
\hline Iris Images (B) & 965 & $96.5 \%$ \\
\hline Iris Images (C) & 880 & $88 \%$ \\
\hline
\end{tabular}


Table 2 indicates that the method is nearly device independent, which maybe profits from feature normalization and down sampling. The recognition rate decreased sharply when users wear glasses and the reason is reflection. When users wear glasses, there may be much reflection in images and the reflection changes the gray level distribution. Indeed, the accuracy is measured by eye-view, so the results are subjective. Some examples are shown in Fig.5, where white box indicates the position of an iris.

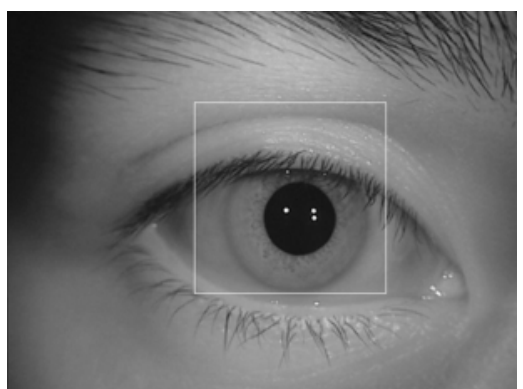

(a)

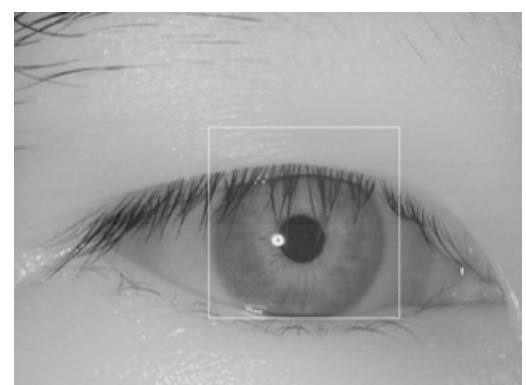

(b)

Fig. 5. Detection results (a) sensor 1 (b) sensor 2

To select proper number of layers, the following experiment is done. We count the number (percentage p) of images that can pass (at most) $x$ layers and draw the histogram $\mathrm{p}$ vs $\mathrm{x}$ in Fig.6. In Fig.6, $\mathrm{x}$-axis and y-axis denote layer number and image percentage, respectively. Black bar denotes positive samples and cyan bar denotes negative samples. It can be seen that most negative samples can be rejected using 16 layers; however, in real application we use 24 layers because there are many face images with closed or half-closed eyes can pass 23 or 24 layers.

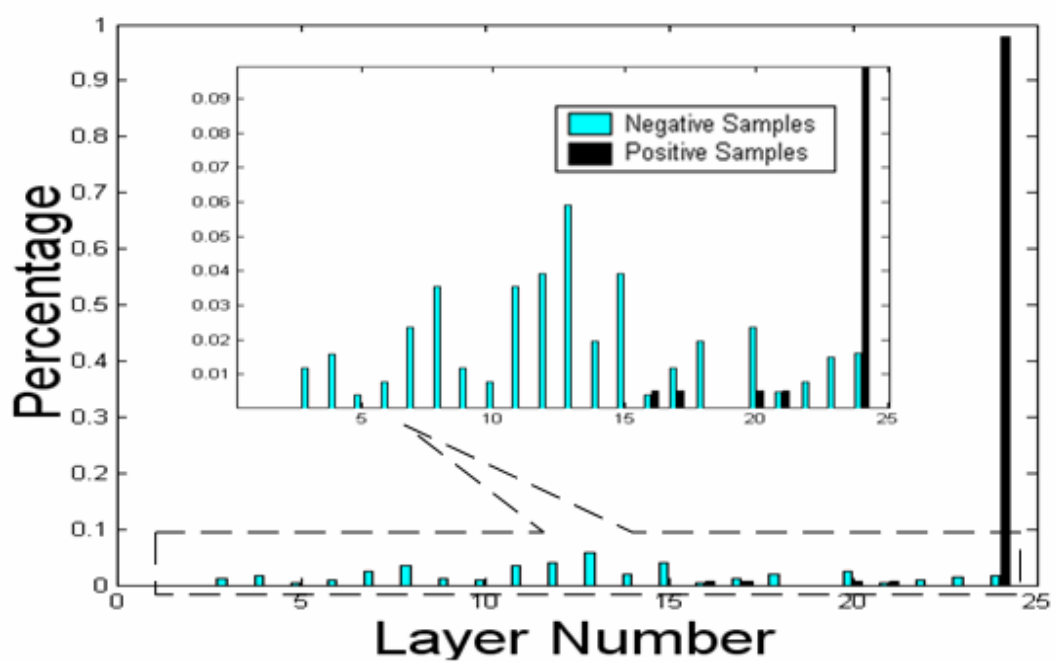

Fig. 6. Sample distribution vs layer number 


\section{Conclusions}

The authors propose an iris detection method based on iris structure characterized by gray value distribution. There are 2 key contributions in this paper. First, it extracts 4 types of features to characterize iris structure. Last, the performance of the proposed method is promising in terms of high speed, accuracy and device independence. The method is fast and, therefore, it can be used in real time systems and can search an image exhaustively. The high recognition rate indicates that structure information is very efficient in iris detection. Moreover, the method is device independent, so when it is used in a new device, it does not need new training. In the future, the authors will focus on the method to overcome glasses reflection and collect more negative samples.

\section{Acknowledgements}

This work is funded by research grants from the National Basic Research Program (Grant No. 2004CB318110), the Natural Science Foundation of China (Grant No. 60335010, 60121302, 60275003, 60332010, 69825105), the Chinese National HiTech R\&D Program (Grant No. 2001AA114180) and the CAS. The authors should also present their thanks to Dr. Chenghua Xu for his help and useful discussions.

\section{References}

1. A.K.Jain, R.M.Bolle and S.Pankanti, Eds., Biometrics: Personal Identification in a Networked Society, Norwell, MA: Kluwer, 1999.

2. J.Daugman, "High Confidence Visual Recognition of Persons by a Test of Statistical Independence”, IEEE Trans. Pattern Analysis and Machine Intelligence, Vol. 15, No.11, pp.1148-1161,1993.

3. R.Wildes, J.Asmuth, et al., “A Machine-vision System for Iris Recognition”, Machine Vision and Applications, Vol.9, pp.1-8, 1996.

4. Seung-In Noh, Kwanghyuk Bae, Yeunggyu Park, Jaihie Kim, “A Novel Method to Extract Features for Iris Recognition System”, LNCS, Volume 2688 / 2003, pp. 862 868, August 2003.

5. L. Ma, T. Tan, Y. Wang and D. Zhang, "Personal Identification Based on Iris Texture Analysis”, IEEE Trans. on Pattern Analysis and Machine Intelligence, Vol. 25, No. 12, pp.1519-1533, 2003.

6. Jiali Cui, Li Ma, Yunhong Wang, Tieniu Tan and Zhenan Sun, “An Appearance-based Method for Iris Detection”, the 6th Asian Conference on Computer Vision (ACCV), Vol. 2, pp. 1091-1096, 2004, Jeju Korea.

7. Paul Viola and Michael Jones, "Robust Real-time Object Detection”, International Journal of Computer Vision 57(2), 137-154, 2004. 